\title{
CHARLIER SPACES OF ENTIRE FUNCTIONS
}

\author{
LOUIS DE BRANGES AND DAVID TRUTT
}

The paper is concerned with examples of Hilbert spaces whose elements are entire functions and which have these properties:

(H1) Whenever $F(z)$ is in the space and has a nonreal zero $w$, the function $F(z)(z-\bar{w}) /(z-w)$ belongs to the space and has the same norm as $F(z)$.

$(\mathrm{H} 2)$ For each nonreal number $w$, the linear functional defined on the space by $F(z) \rightarrow F(w)$ is continuous.

(H3) The function $F^{*}(z)=\bar{F}(\bar{z})$ belongs to the space whenever $F(z)$ belongs to the space, and it always has the same norm as $F(z)$.

The theory of these spaces is related to the theory of entire functions $E(z)$ which satisfy the inequality

$$
|E(x-i y)|<|E(x+i y)|
$$

for $y>0$. If $E(z)$ is such a function, we write $E(z)=A(z)-i B(z)$ where $A(z)$ and $B(z)$ are entire functions which are real for real $z$, and

$$
K(w, z)=[B(z) \bar{A}(w)-A(z) \bar{B}(w)] /[\pi(z-\bar{w})] .
$$

Let $\mathfrak{H C}(E)$ be the set of entire functions $F(z)$ such that

$$
\|F\|^{2}=\int_{-\infty}^{+\infty}|F(t) / E(t)|^{2} d t<\infty
$$

and such that

$$
|F(z)|^{2} \leqq\|F\|^{2} K(z, z)
$$

for all complex $z$. Then $\mathfrak{F}(E)$ is a Hilbert space of entire functions which satisfies the axioms (H1), (H2), and (H3). For each complex number $w, K(w, z)$ belongs to $\mathscr{H}(E)$ as a function of $z$, and the identity

$$
F(w)=\langle F(t), K(w, t)\rangle
$$

holds for all elements $F(z)$ of $\mathfrak{H C}(E)$. A Hilbert space, whose elements are entire functions, which satisfies the axioms (H1), (H2), and (H3), and which contains a nonzero element is equal isometrically to a space $\mathfrak{F C}(E)$.

The spaces now studied are finite dimensional spaces related to Charlier's orthogonal polynomials. They are characterized by an

Received by the editors September 4, 1967. 
identity involving a positive parameter $a$. The identity implies a recurrence relation for the defining functions $A(z)$ and $B(z)$ of the space.

TheOREM 1. Let $\operatorname{HC}(E)$ be a given space such that $E(z)$ has a nonzero value at the origin, and let $a$ be a given positive number. Assume that the functions $z[F(z)-F(z-1)]$ and $F(z+1)-F(z)$ belong to the space whenever $F(z)$ belongs to the space, and that the identity

$$
\begin{aligned}
\langle t[F(t)- & \left.F(t-1)]-a^{2}[F(t+1)-F(t)], G(t)\right\rangle \\
& =\left\langle F(t), t[G(t)-G(t-1)]-a^{2}[G(t+1)-G(t)]\right\rangle
\end{aligned}
$$

holds for all elements $F(z)$ and $G(z)$ of the space. Then there exist real numbers $u_{+}, v_{+}, u_{-}, v_{-}$such that the functions

$$
S_{+}(z)=A(z) u_{+}+B(z) v_{+} \text {and } S_{-}(z)=A(z) u_{-}+B(z) v_{-}
$$

are linearly independent and satisfy the recurrence relations

$$
\begin{aligned}
\lambda_{+} S_{+}(z) & =z\left[S_{+}(z)-S_{+}(z-1)\right]-a^{2}\left[S_{+}(z+1)-S_{+}(z)\right] \\
\lambda_{-} S_{-}(z) & =z\left[S_{-}(z)-S_{-}(z-1)\right]-a^{2}\left[S_{-}(z+1)-S_{-}(z)\right] \\
\lambda_{+} S_{-}(z) & =a S_{+}(z+1)-a S_{+}(z), \\
S_{+}(z) & =a^{-1} z S_{-}(z-1)-a S_{-}(z)
\end{aligned}
$$

for some real numbers $\lambda_{+}$and $\lambda_{-}$such that $\lambda_{+}=1+\lambda_{-}$.

Kummer's confluent hypergeometric function

$$
F(a ; c ; z)=1+\frac{a}{1 ! c} z+\frac{a(a+1)}{2 ! c(c+1)} z^{2}+\cdots
$$

is used to construct spaces satisfying the hypotheses of Theorem 1.

Theorem 2. If $a>0$ is given, then the polynomials $\Phi_{n}(z)$ defined by

$$
\Phi_{n}(z)=(-a)^{-n}[\Gamma(n-z) / \Gamma(-z)] F\left(-n ; 1+z-n ; a^{2}\right)
$$

are real for real $z$ and satisfy the identities

$$
\begin{aligned}
n \Phi_{n}(z) & =z\left[\Phi_{n}(z)-\Phi_{n}(z-1)\right]-a^{2}\left[\Phi_{n}(z+1)-\Phi_{n}(z)\right] \\
n \Phi_{n-1}(z) & =a \Phi_{n}(z+1)-a \Phi_{n}(z), \\
\Phi_{n+1}(z) & =a^{-1} z \Phi_{n}(z-1)-a \Phi_{n}(z), \\
z \Phi_{n}(z) & =a n \Phi_{n-1}(z)+\left(n+a^{2}\right) \Phi_{n}(z)+a \Phi_{n+1}(z) .
\end{aligned}
$$

There exist spaces $\mathfrak{H}\left(E_{n}\right), n=1,2,3, \cdots$, satisfying the hypotheses of Theorem 1, such that $\mathfrak{H C}\left(E_{n}\right)$ is contained isometrically in $\operatorname{HC}\left(E_{n+1}\right)$ 
for every $n$, such that $\Phi_{0}(z)$ spans $\mathcal{H}\left(E_{1}\right)$, and such that $\Phi_{n}(z)$ spans the orthogonal complement of $\mathfrak{H C}\left(E_{n}\right)$ in $\mathfrak{H C}\left(E_{n+1}\right)$ for every $n>0$. The spaces can be chosen so that $\left\|\Phi_{n}(t)\right\|^{2}=\Gamma(1+n)$ for every $n$. The identity

$$
e^{a^{2}}\|F(t)\|^{2}=\sum_{n=0}^{\infty}|F(n)|^{2} a^{2 n} / \Gamma(1+n)
$$

then holds for every polynomial $F(z)$.

These are essentially all the spaces which satisfy the hypotheses of Theorem 1.

THEOREM 3. If $\mathfrak{H}(E)$ is a space which satisfies the hypotheses of Theorem 1, then there exists an entire function $S(z)$ which is real for real $z$, has only real zeros, and is periodic of period one, and there exists an index $r$ in Theorem 2 such that the transformation $F(z) \rightarrow S(z) F(z)$ is an isometry of $\mathfrak{H C}\left(E_{r}\right)$ onto $\mathfrak{H C}(E)$.

These spaces, like those of previous work [2], are related to generalized spaces of square summable power series. Let $a$ and $c$ be numbers such that the coefficients of Kummer's series $F(a ; c ; z)$ are all positive. By $\mathrm{e}(a ; c ; z)$ we mean the Hilbert space of power series $f(z)=\sum a_{n} z^{n}$ with complex coefficients such that

$$
\|f(z)\|^{2}=\left|a_{0}\right|^{2}+\frac{1 ! c}{a}\left|a_{1}\right|^{2}+\frac{2 ! c(c+1)}{a(a+1)}\left|a_{2}\right|^{2}+\cdots<\infty .
$$

The series which belong to $\mathfrak{C}(a ; c ; z)$ converge in the complex plane and represent entire functions. The series $F(a ; c ; \bar{v} z)$ belongs to the space for all complex numbers $w$, and the identity

$$
f(w)=\langle f(z), F(a ; c ; \bar{w} z)\rangle
$$

holds for every element $f(z)$ of the space.

THEOREM 4. In Theorem 2 if $f(z)=\sum a_{n} z^{n}$ is a polynomial of degree less than $r$, then its eigentransform $F(z)=\sum a_{n} \Phi_{n}(z)$ belongs to $\operatorname{HC}\left(E_{r}\right)$ and

$$
\int_{-\infty}^{+\infty}\left|F(t) / E_{r}(t)\right|^{2} d t=\|f(z)\|^{2}
$$

where the norm of $f(z)$ is taken in $\mathrm{C}(1 ; 1 ; z)$. Every element of $\operatorname{HC}\left(E_{r}\right)$ is of this form. The identity

$$
\Gamma(z) F(-z)=\int_{0}^{\infty} f(-a-t / a) e^{-t} t^{z-1} d t
$$


holds for $x>0$ whenever $f(z)$ is a polynomial and $F(z)$ is its eigentransform. Let $f(z)$ and $g(z)$ be polynomials, and let $F(z)$ and $G(z)$ be their eigentransforms. The condition

$$
G(z)=z[F(z)-F(z-1)]-a^{2}[F(z+1)-F(z)]
$$

is necessary and sufficient that $g(z)=z f^{\prime}(z)$. The condition $G(z)$ $=a F(z+1)-a F(z)$ is necessary and sufficient that $g(z)=f^{\prime}(z)$. The condition $G(z)=a^{-1} z F(z-1)-a F(z)$ is necessary and sufficient that $g(z)=z f(z)$. The condition $G(z)=z F(z)$ is necessary and sufficient that $g(z)=(z+a) f^{\prime}(z)+a(z+a) f(z)$.

Proof of Theorem 1. Let $L_{+}, L_{-}$, and $D$ be the transformations on entire functions defined by $D: F(z) \rightarrow G(z)$ if

$$
G(z)=z[F(z)-F(z-1)]-a^{2}[F(z+1)-F(z)],
$$

$L_{-}: F(z) \rightarrow G(z)$ if

$$
G(z)=a F(z+1)-a F(z),
$$

and $L_{+}: F(z) \rightarrow G(z)$ if

$$
G(z)=a^{-1} z F(z-1)-a F(z) .
$$

A straightforward calculation will show that the commutator identities

$$
D L_{-}-L_{-} D=-L_{-}, \quad D L_{+}-L_{+} D=L_{+}, \quad L_{-} L_{+}-L_{+} L_{-}=1,
$$

are satisfied. By hypothesis the restriction of $D$ to the space is a selfadjoint transformation in the space. Since $D$ is everywhere defined in the space, it is bounded. The hypotheses also imply that $L_{-}$takes the space into itself. Since the restriction of $L_{-}$to the space has a closed graph, it is bounded. If $F(z)$ belongs to the domain of multiplication by $z$ and if $D: F(z) \rightarrow G(z)$, then

$$
D: z F(z) \rightarrow z G(z)+z F(z-1)-a^{2} F(z+1) .
$$

It follows that the identity

$$
\left\langle t F(t-1)-a^{2} F(t+1), G(t)\right\rangle=-\left\langle F(t), t G(t-1)-a^{2} G(t+1)\right\rangle
$$

holds whenever $F(z)$ and $G(z)$ belong to the domain of multiplication by $z$ in the space. Since $D$ is selfadjoint, the identity

$$
\langle t F(t-1), G(t)\rangle=a^{2}\langle F(t), G(t+1)\rangle
$$

holds whenever $F(z)$ and $G(z)$ belong to the domain of multiplication by $z$ in the space. As in the proof of Theorem 1 of [2], this implies 
that $L_{+}$acts as a bounded transformation on the domain of multiplication by $z$. Since the action of $D+a L_{+}+a L_{-}$coincides with multiplication by $z$, multiplication by $z$ is a bounded transformation in the space. An argument in the proof of Theorem 1 of [2] will show that the space is finite dimensional.

Let $r$ be the dimension of the space. Since we assume that $E(z)$ has a nonzero value at the origin, there exists an element of the space which has a nonzero value at the origin. Since the transformation $F(z) \rightarrow z[F(z)-F(z-1)]$ does not take the space onto itself, it has a nonzero kernel. It follows that there exists a nonzero element $S(z)$ of the space which is periodic of period one. Since $z[F(z+1)-F(z)]$ belongs to the space whenever $F(z)$ belongs to the space, and since $E(z)$ has a nonzero value at the origin, the functions $F(z+1)-F(z)$ and $F(z+1)$ belong to the space whenever $F(z)$ belongs to the space. Since the space is finite dimensional, there exists no zero $w$ of $S(z)$ such that $S(z) /(z+n-w)$ belongs to the space for every $n=0,1$, $2, \ldots$. It follows that there exists no zero $w$ of $S(z)$ such that $S(z) /(z-w)$ belongs to the space. By Problem 88 of [1], $S(z)$ and $S^{*}(z)$ are linearly dependent, and the elements of the space are the entire functions $F(z)$ such that $F(z) / S(z)$ is a polynomial of degree less than $r$. We assume that $S(z)$ is chosen of norm one and real for real $z$.

Let $S_{0}(z), S_{1}(z), S_{2}(z), \cdots$ be the entire functions defined inductively by $S_{0}(z)=S(z)$ and

$$
L_{+}: S_{n}(z) \rightarrow S_{n+1}(z) .
$$

The commutator identities imply that

$$
D: S_{n}(z) \rightarrow n S_{n}(z)
$$

for every $n$ and that

$$
L_{-}: S_{n}(z) \rightarrow n S_{n-1}(z)
$$

for every $n>0$. It follows that the identity

$$
z S_{n}(z)=a n S_{n-1}(z)+\left(n+a^{2}\right) S_{n}(z)+a S_{n+1}(z)
$$

holds for $n>0$, and for $n=0$ with the term in $S_{n-1}(z)$ omitted. It is clear that $S_{n}(z) / S(z)$ is a polynomial of degree $n$. So $S_{n}(z)$ belongs to $\mathfrak{H C}\left(E_{r}\right)$ when $n<r$. The functions $S_{0}(z), \cdots, S_{r-1}(z)$ are orthogonal in $\mathfrak{F C}\left(E_{r}\right)$ since they are eigenfunctions of a selfadjoint operator for distinct eigenvalues. Since multiplication by $z$ is a symmetric transformation, the identity

$$
\left\langle t S_{n-1}(t), S_{n}(t)\right\rangle=\left\langle S_{n-1}(t), t S_{n}(t)\right\rangle
$$


holds when $0<n<r$. It follows that $\left\|S_{n}(t)\right\|^{2}=n\left\|S_{n-1}(t)\right\|^{2}$. Since we assume that $\left\|S_{0}(t)\right\|=1$, we can conclude that $\left\|S_{n}(t)\right\|^{2}=\Gamma(1+n)$.

As in the proof of Theorem 1 of [2], there exists a space $\mathcal{H C}\left(E_{r+1}\right)$, satisfying the hypotheses of Theorem 1 , which contains $\mathfrak{H C}\left(E_{r}\right)$ isometrically, such that $S_{r}(z)$ spans the orthogonal complement of $\mathfrak{F C}\left(E_{r}\right)$ in $\mathfrak{F C}\left(E_{r+1}\right)$. The theorem now follows from Theorem 23 and Problem 87 of [1].

Proof of Theorem 2. It is clear from the definition of Kummer's series that $\Phi_{n}(z)$ is a polynomial of degree $n$ which is real for real $z$. The stated identities for $\Phi_{n}(z)$ follow from the well-known relations between contiguous hypergeometric series, Erdélyi [3]. Consider the unique inner product on polynomials with respect to which the functions $\Phi_{n}(z)$ are an orthogonal set and $\left\|\Phi_{n}(t)\right\|^{2}=\Gamma(1+n)$ for every $n$. Define $L_{+}, L_{-}$, and $D$ as in the proof of Theorem 1. It is easily verified that the identities

$$
\begin{aligned}
\langle D F, G\rangle & =\langle F, D G\rangle, \\
\left\langle L_{+} F, G\right\rangle & =\left\langle F, L_{-} G\right\rangle, \\
\langle t F(t), G(t)\rangle & =\langle F(t), t G(t)\rangle
\end{aligned}
$$

hold for all polynomials $F(z)$ and $G(z)$. For every $r=1,2,3, \cdots$, the polynomials of degree less than $r$ are a Hilbert space of entire functions which satisfies the axioms $(\mathrm{H} 1),(\mathrm{H} 2)$, and $(\mathrm{H} 3)$. By Theorem 23 of [1], the space is equal isometrically to a space $\mathscr{H}\left(E_{r}\right)$. The function $E_{r}(z)$ has a nonzero value at the origin since the space contains a constant function which has a nonzero value at the origin. The polynomials $z[F(z)-F(z-1)]$ and $F(z+1)-F(z)$ belong to the space whenever $F(z)$ belongs to the space since their degrees do not exceed the degree of $F(z)$. The restriction of $D$ to the space is selfadjoint since the space admits an orthogonal basis of eigenfunctions of $D$ corresponding to real eigenvalues. From this we see that $\mathfrak{H C}\left(E_{r}\right)$ satisfies the hypotheses of Theorem 1 for every $r$.

To complete the proof of the theorem, we consider a new inner product on polynomials defined by

$$
\langle F(t), G(t)\rangle_{1}=\sum_{n=0}^{\infty} F(n) \bar{G}(n) a^{2 n} / \Gamma(1+n) .
$$

It is easily verified that the identity

$$
\langle t F(t-1), G(t)\rangle_{1}=a^{2}\langle F(t), G(t+1)\rangle_{1}
$$

holds for all polynomials $F(z)$ and $G(z)$. It follows that the identities

$$
\langle D F, G\rangle_{1}=\langle F, D G\rangle_{1}, \quad\left\langle L_{+} F, G\right\rangle_{1}=\left\langle F, L_{-} G\right\rangle_{1},
$$


hold for all polynomials $F(z)$ and $G(z)$. The proof of Theorem 1 will show that

$$
\langle F(t), G(t)\rangle_{1}=\kappa\langle F(t), G(t)\rangle
$$

for all polynomials $F(z)$ and $G(z)$, where $\kappa$ is a constant. When $F(z)=G(z)=1$, we obtain

$$
\kappa=\sum_{n=1}^{\infty} a^{2 n} / \Gamma(1+n)=e^{a^{2}} .
$$

Proof of Theorem 3. This more general result follows from the above proofs of Theorems 1 and 2.

Proof of Theorem 4. The theorem follows by a routine calculation once it is known that the formula

$$
\Gamma(z) \Phi_{n}(-z)=\int_{0}^{\infty}(-a-t / a)^{n} e^{-t} t^{z-1} d t
$$

holds for every $n$ when $x>0$. The formula is true when $n=0$ by the definition of the gamma function. A straightforward calculation will show that the functions defined by this integral formula satisfy the recurrence relations of Theorem 2 . These functions must therefore coincide with the functions of Theorem 2 .

\section{REFERENCES}

1. L. de Branges, Hilbert spaces of entire functions, Prentice-Hall, Englewood Cliffs, N. J., 1968.

2. L. de Branges and D. Trutt, Meixner and Pollaczek spaces of entire functions, J. Math. Anal. Appl. 22 (1968), 12-24.

3. A. Erdélyi, W. Magnus, F. Oberhettinger and F. G. Tricomi, Higher transcendental functions, Vol. 1, McGraw-Hill, New York, 1953.

Purdue University and

LEHIGH UNIVERSITY 